\title{
A Study on Motion and Position Using AHRS Based on Inertial Measurement Unit in Virtual Reality Environments
}

\author{
Am-Suk Oh \\ Dept. of Digital Media Engineering, Tongmyong University, Busan, Korea \\ asoh@tu.ac.kr
}

\begin{abstract}
This thesis is about improving the accuracy of motion and location for enhancing user immersion in experience type VR environment about position tracking technique. To achieve this, Motion Tracking \& Localization (MTL) estimated by utilizing 9axis Inertial Measurement Unit (IMU) based Attitude \& Heading Reference System (AHRS). Moreover, MTL device design, AHRS based motion tracking technique and position estimation technique considering experience type VR environment suggested. Ultimately, test bed construction of multiple AHRS based position tracking and performance evaluation are processed.
\end{abstract}

Keywords: Virtual Reality, Motion Tracking, Localization, Attitude \& Heading Reference System, Inertial Measurement Unit

\section{Introduction}

In terms of the virtual reality, recently, device and gaming software were the major markets through the development of ICT-related techniques. However, it is expected that the software market would lead the growth of the virtual reality market as the various contents and the software have enlarged [1-2].

Although people could access to VR contents through HMD supply, many individuals experience maladjustment and lowering immersion of VR. Such phenomenon occurs mostly because the movements delivered through VR video do not match with actual motion extent or response [3-4].

To maximize VR effect recently, therefore, experience type VR system which enables user to interact by feeling situations or environments in virtual space and strengthens immersion or sense of reality by utilizing existing physical space or equipment has been developed [5-6].

Virtual reality equipment, the hardware that interconnect user and virtual space consists of hardware such as HMD, controller and sensor. However, users require capability of interaction between objects in virtual space just like in reality by recognizing body movements that move according to willing, thus, expressing in virtual space [7-8].

Motion expression is limited with controllers only such as HMD, gloves, vests and gun and whole body motion tracking technique that can accurately express and grasp the user's intention is needed for the implementation of user experience type VR. Apart from motion tracking, moreover, location tracking with high accuracy is required because many dynamic objects have to interact together in real time [9-10].

Generally, GPS used as location recognition technique for walkers. GPS, satellitebased ratio wave navigating equipment, provides relatively accurate and absolute position. However, it does not work separately in GPS signal shaded area such as indoor or tunnel [11-12]. In order to resolve it, position recognition system fingerprinting method that utilize WiFi and indoor positioning technique that utilize trilateration technique is

Received (April 16, 2018), Review Result (July 3, 2018), Accepted (July 25, 2018) 
getting attention particularly and the research about accuracy and efficiency that utilize such positioning techniques are under the developments [13-14].

\section{Relative Work}

\subsection{Virtual Reality}

Virtual reality is the technique that stimulates five senses (sight, hearing, smell, taste, touch) of the body that cannot obtained in reality using artificial techniques so that one experiences as if it is real. Recently, virtual reality techniques are spreading thanks to the development of ICT-related techniques and the change in the contents manufacturing environment [15-16].

The main parts of the early virtual reality markets were HMD (head-mounted display) device and gaming software. Since 2017, however, it expected that contents and software markets lead the growth of the virtual reality markets as various contents and software have enlarged. The VR market scale of the world in 2015 was 2.3 billion dollars and it increased by $65.2 \%$, to 3.8 billion dollars in 2016 and it expected to reach 5.2 billion dollars in 2018. The domestic VR market scale was approximately 9.6 billion dollars in 2015 predicted to grow by $42.4 \%, 13.7$ billion dollars in 2016 and expected to reach 50.7 billion dollars in 2020 [17-18]. Figure 1 and Figure 2 show this market scale.

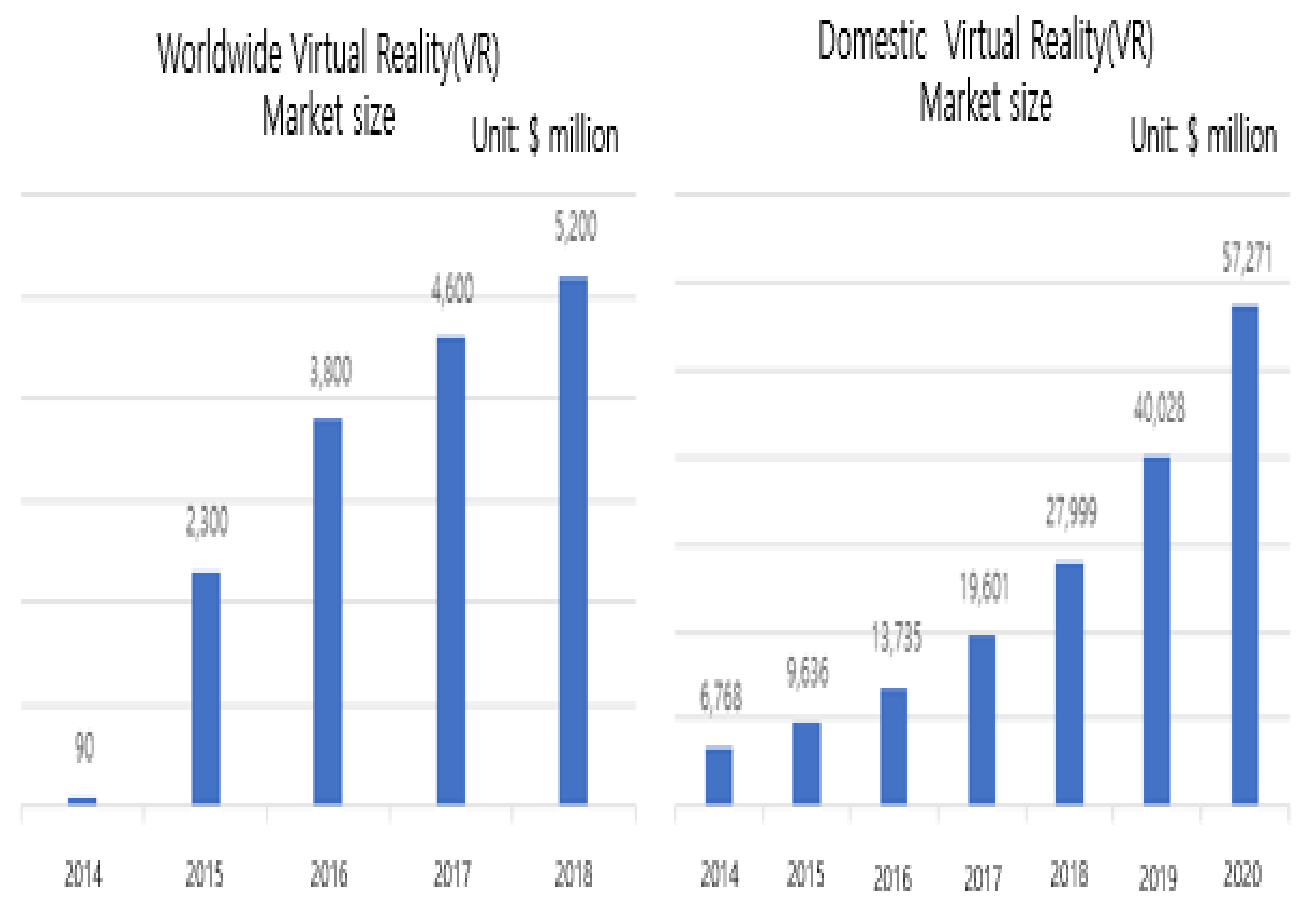

Figure 1. Domestic and Foreign Virtual Reality Market Size 

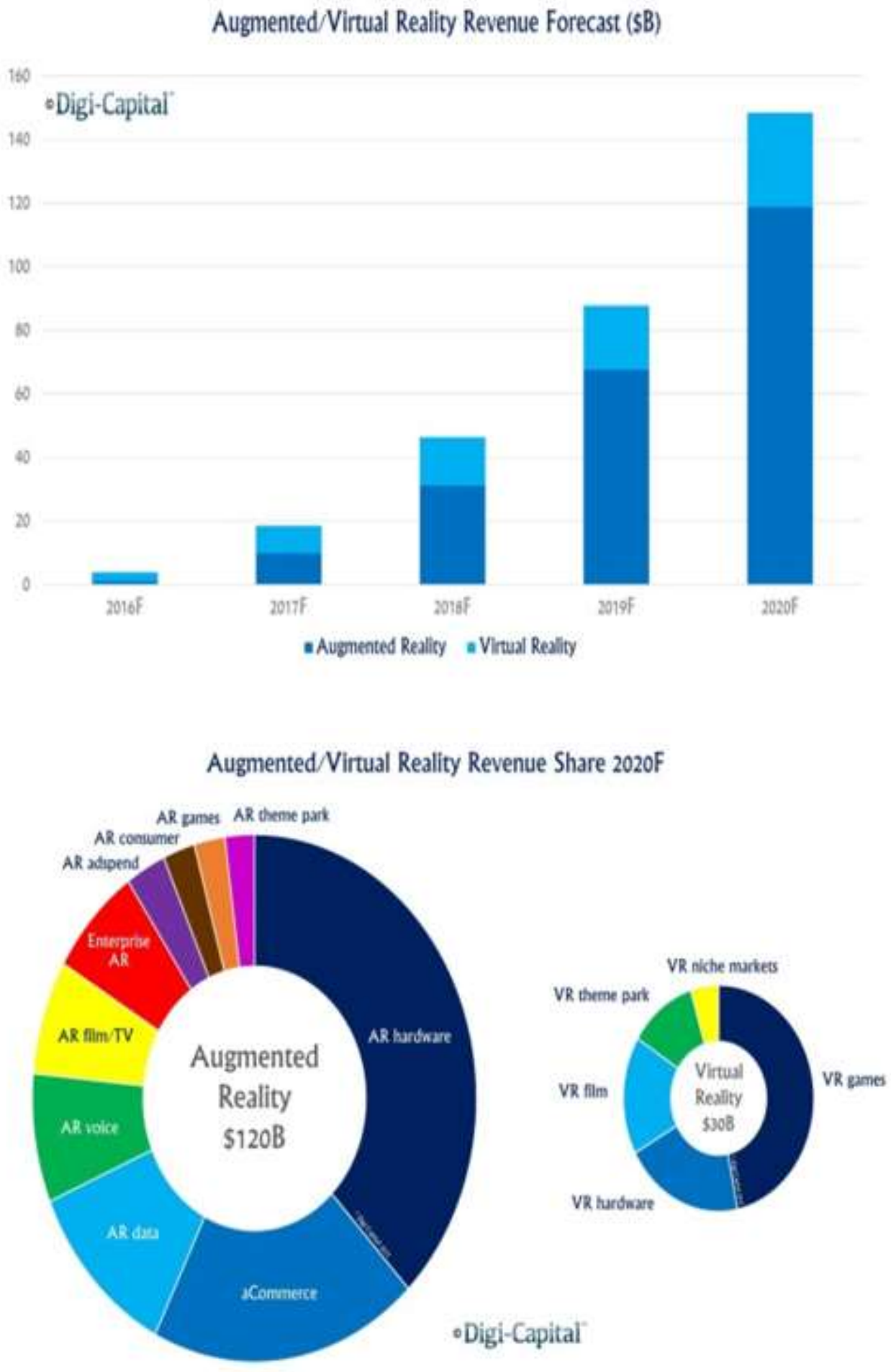

Figure 2. Augmented Reality \& Virtual Reality Comparison [17] [18]

\subsection{Virtual Reality Paradigm}

In spite of the experience of VR contents through HMD supply, quite a few people experience about lowering immersion and maladjustment of VR. Such phenomenon occur since the motion delivered through VR video does not match with the actual motion extent or reaction. In order to maximize VR effect, accordingly, experience type VR system that enables the user to interact by feeling situations or environments in virtual 
space through their senses, and that enhances the immersion or presence of VR using existing physical space or equipment is under development. To implement the experience type VR system, the function that outputs videos in real time according to the user's motion is fundamentally required. Furthermore, stereophonic sound that controls the direction of sound according to the movement other than HMD, and the sensor or motion recognition technique that recognizes the interactions and the movements among the users and the virtual objects need to be complex utilized.

\subsection{Virtual Reality Application}

VR originated from the flow that fuses with diverse fundamental industry such as CPND (Content-Platform-Network-Device) based broadcasting, movie, communication, gaming, manufacturing, medical treatment, home appliances, mobile, cars, and robots. Accordingly, the results of this research can grafted to various field, and the new creating value expected through this.

Field of medical treatment: enables remote surgery using robot equipment and conveniently educates medical doctors in surgery, diagnosis, and psychotherapy.

Field of the military: applied to diverse solutions such as parachute, air crafting control, finding target, and battle simulations.

Field of the gaming: VR already actively commercialized in gaming fields. one can experience VR only with sight and hearing and will be able to control using five senses in the future.

Field of education: expected to improve the efficiency by experiencing human organs and physical phenomenon through VR in various education fields such as science, medical treatment or history.
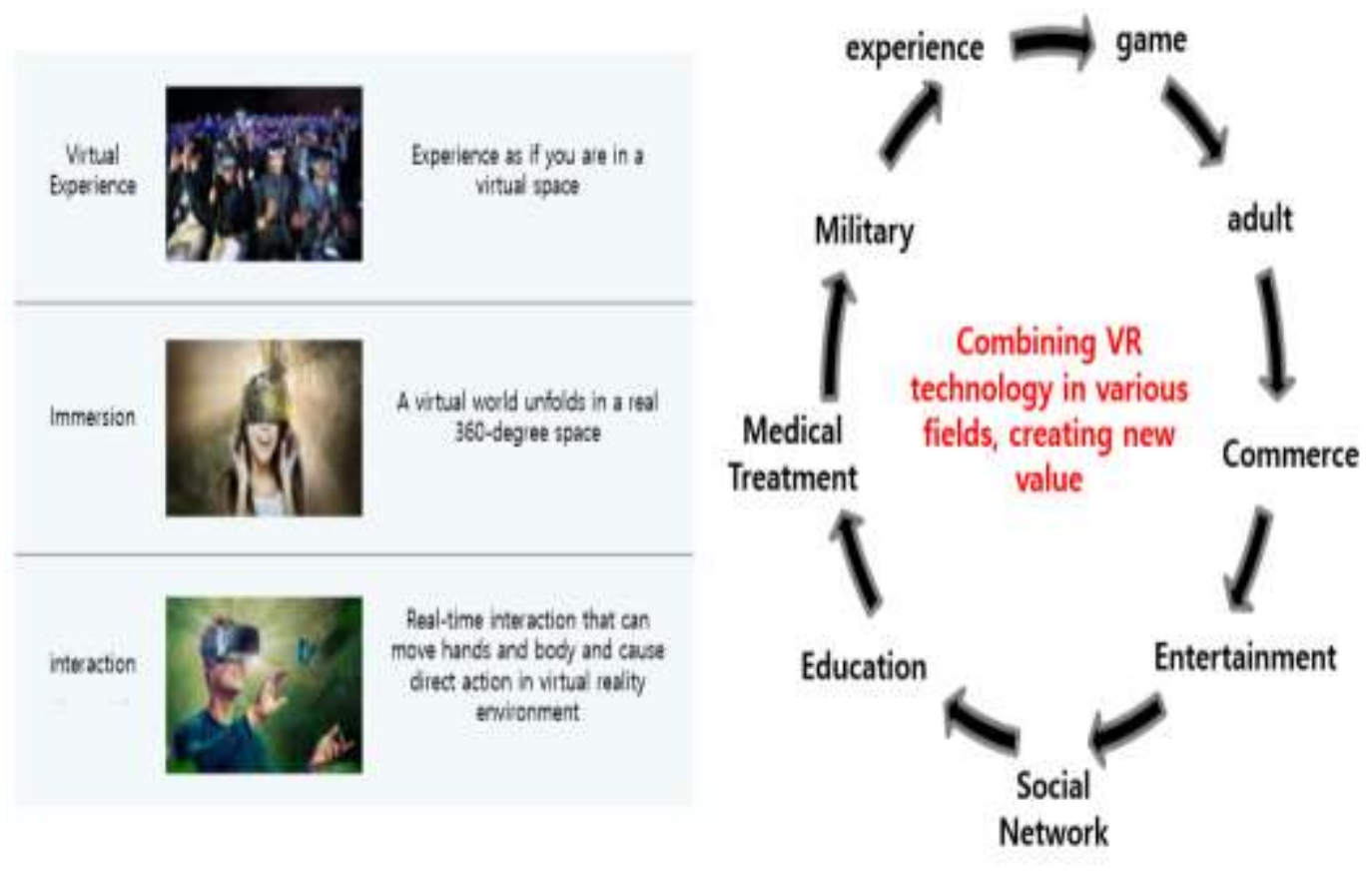

Figure 3. Application Field 


\section{Design of MTL Device}

In VR field, currently, research and development are in the process mostly based on HMD and utilizes asynchronous communication and calculation for real time data handling such as screen switch and calculation. In asynchronous serial communication, however, UART causes overhead by sync and requires complex hardware controller for data sending and receiving, thus it is not appropriate for communicating method for multiple AHRS-based position tracking system. Accordingly, this paper designs communication module that utilizes SPI, which is a typical synchronous serial communicating method to improve the transmission efficiency.

In VR, motion to photon latency (MTP), the time gap between motion and screen switch, should minimized for the sake of user's immersion.

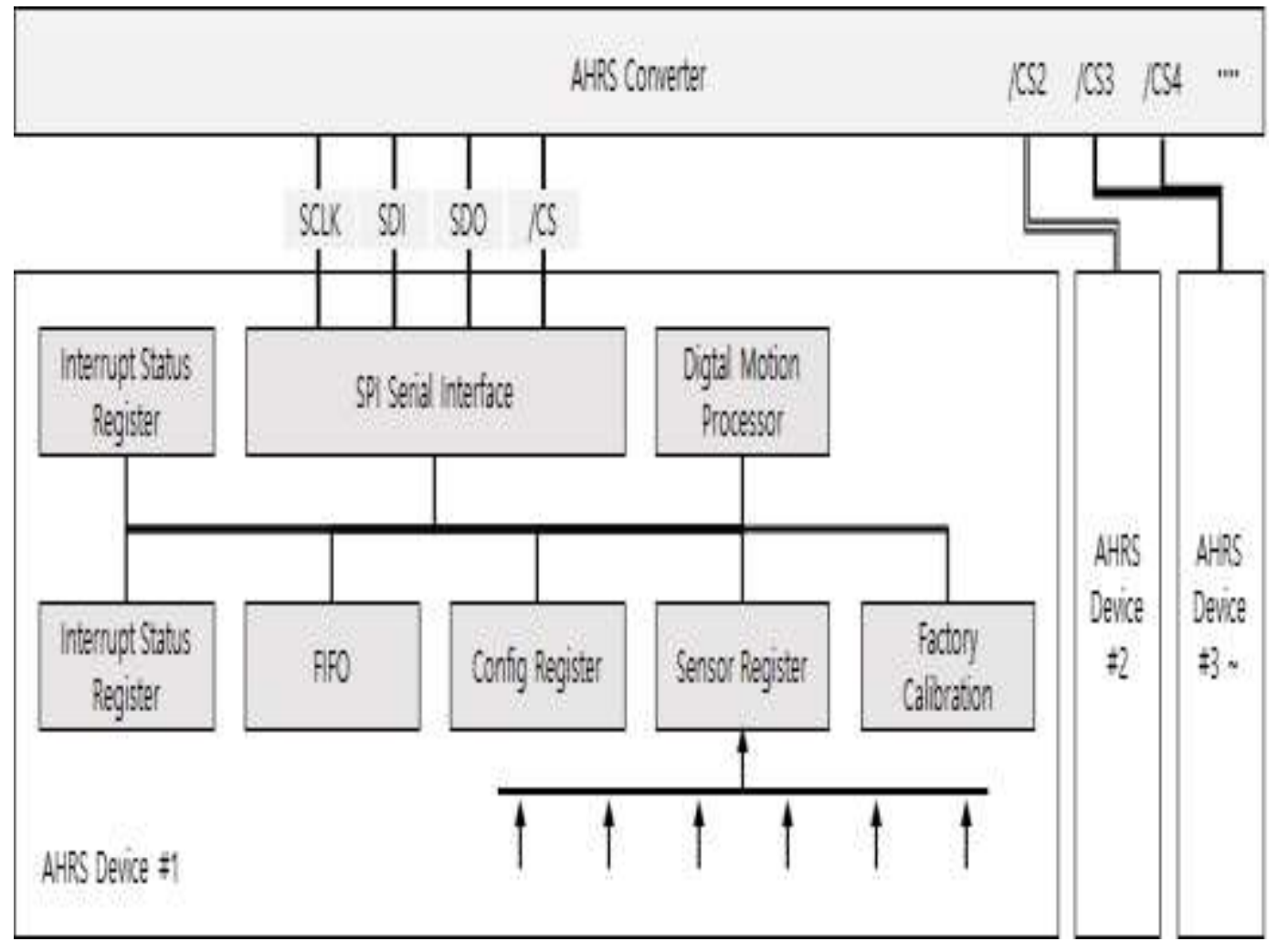

Figure 4. AHRS Device Connection Configuration

In experience type VR system, motion recognition for expressing the motion of user or partner works as major technique. Commonly used motion recognition method at the moment tracks user's motion by estimating the position of joints, defined according to the human body through depth camera, infra, or photodiode in VR infra. However, such methods cause error in motion estimation when visual obstacles exist in between infra and MTL device and trembling phenomenon could occur even in stationary state at hands or feet that belong to the end of the position estimation information of joints.

Accordingly, this paper suggests the method that estimates the user's motion based on the 9-axis movement information measured from each AHRS device, attaching several AHRS devices onto the body that can collect link information of joints so coordinates system can defined. Suggested position estimation of the user detects the position of link coordinates by calculating the position of each joint through the combination of relative position information of body coordinate system relative to local coordinate system of link equipped with AHRS device. 


\section{Motion Tracking Method and Moving Path Estimation}

\subsection{Motion Tracking Method}

Typical methods to minimize the error during non-linear situation such as dynamic movement are Kalman filter and unscented Kalman filter, which are Bayesian filter based. However, such Kalman filters have disadvantages that they are hard to control and have many variables to regulate in terms of position expression that has unique Euler angle or angle-axis expression due to the constraint rotation matrix has. For the improvement of real time and accuracy of position tracking in VR environment, therefore, this paper researches about position tracking algorithm which is capable of filter setting only with simplified particular conditional variable adjustment, and supports simple calculation such as diagonalization of small unit symmetric matrix based on recursive Bayesian's technique.

In experience type VR system, the motion tracking to express the motion of user or others works as a major technique. The most widely used motion tracking method tracks user's motion by estimating the position of joint defined by the human body through depth camera, infra or photodiode in VR infra. However, such method generates error in estimation when there are visual obstacles between infra and MTL device, and trembling phenomenon could occur during stationary state at estimated position such as hands or feet belong to the end of position estimation information of joints.

We propose about motion estimation algorithm that assumes user motion based on 9axis motion information measured from each AHRS device defining coordinates system, attached on the body is capable of collecting link information of joint.

User motion estimation that this thesis suggests detects the position of link coordinates by calculating the position of each joint through the combination of link information with the relative posture information of body coordinate system relative to local coordinate system of link equipped with AHRS device.

At this stage, displacement compensation of each joint through data filter for the accuracy of motion is required. Therefore, this paper researches about algorithm that extracts joint angle among body segments is biomechanics measured value, and through this, researches about techniques to correct or filter user motion tracking data about position information of each joint.

\subsection{Moving Path Estimation}

The motion tracking technique this paper suggests is capable of lower body motion tracking based on position information through the calculation of displacement of joints. Such AHRS-based position recognition is one of the relative position recognition methods that estimate next position by accumulating position variation from current position.

Although it does not require extra working environment compared to absolute position recognition methods and can quickly update position information or route due to low operation amount, the error in user position variation accumulated over time. Accordingly, this paper researches about position displacement compensation algorithm for the improvement in the error accumulation from relative position recognition method over time, and through this, researches about AHRS-based position recognition and route estimation technique.

In order to guarantee the accuracy in position recognition, quick location update through the combination of relative and absolute position recognition methods is required and accumulated error over time needs to remove through regular location correction. To improve the cumulative error of AHRS-based location recognition technique (relative position recognition), this paper researches about location recognition filtering and correction technique that utilizes bluetooth RSSI-based Fingerprint method that does not require extra calculation and whose distance measurement model is relatively simple 
(absolute position recognition), and through this, the improvement in accuracy of location recognition and route is expected.

\section{Moving Recognition Technology and Position Recognition Technology}

\subsection{Moving Recognition Technology}

Interface technique that tracks body movement of users other than their head or eyes and reflects them to virtual space is required to conduct the function that user interacts with contents objects using their body. The form of gloves commonly used in the past, but it has become more and more sophisticated and it can now track the finger movements. The early VR was only capable of showing $360^{\circ}$ video according to the directions by tracking the direction of head movements using HMD while sitting, but thanks to motion tracking technique and 3D scan technique developments, free movements in real space is now possible.

Figure 5 indicates a person using Oculus Rift (HMD) from VR companies. As shown in Figure 5, detecting the head movement of user through HMD device attached onto the face, the screen moves as the head moves. While existing HMD only showed the screen right in front of the eyes, it highly improved immersion by enhancing viewing angle through camera tracking and optical tracking. Furthermore, it enabled more directly interaction with VR contents through Oculus touch, which is input tool used by both hands.
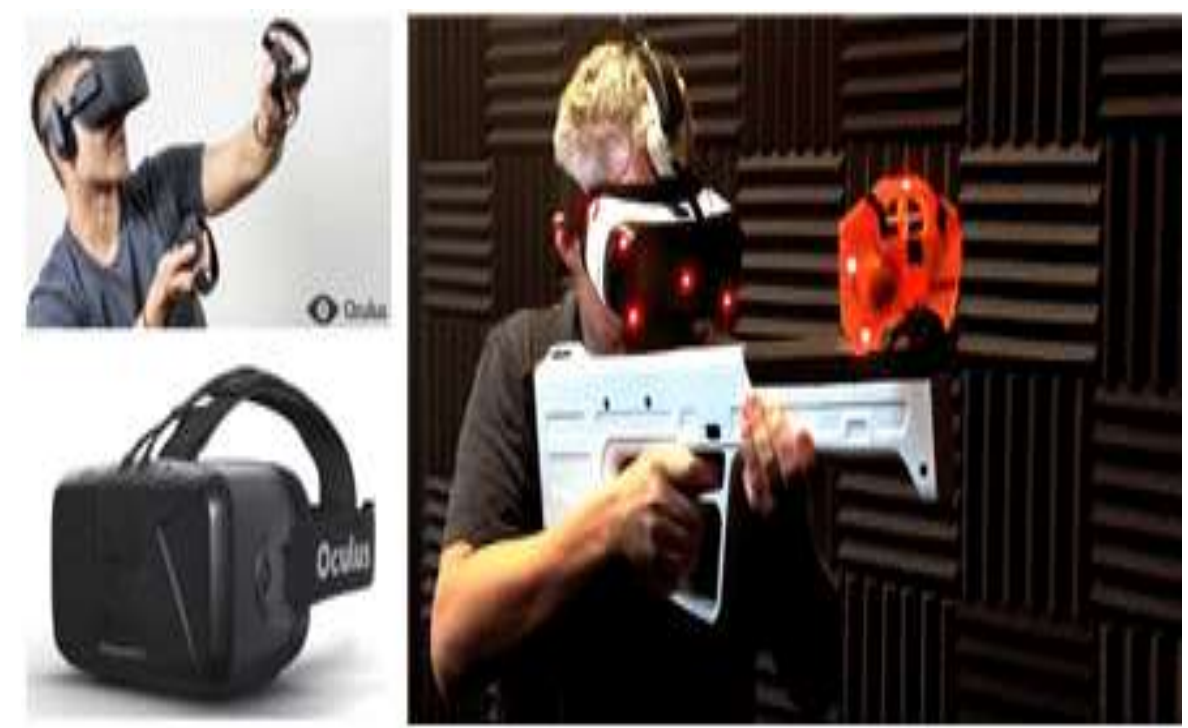

Figure 5. HMD (Oculus Rift) and the Handy Type Controller (Haptic VR Gun)

\subsection{Position Recognition Technology}

Currently used indoor location recognition techniques are WiFi and BLE. In the Figure 6, it shows RSSI measured values of Wi-Fi and BLE within $3.5 \mathrm{~m}$. However, the irregular Fading in Wi-Fi makes recognition harder. 


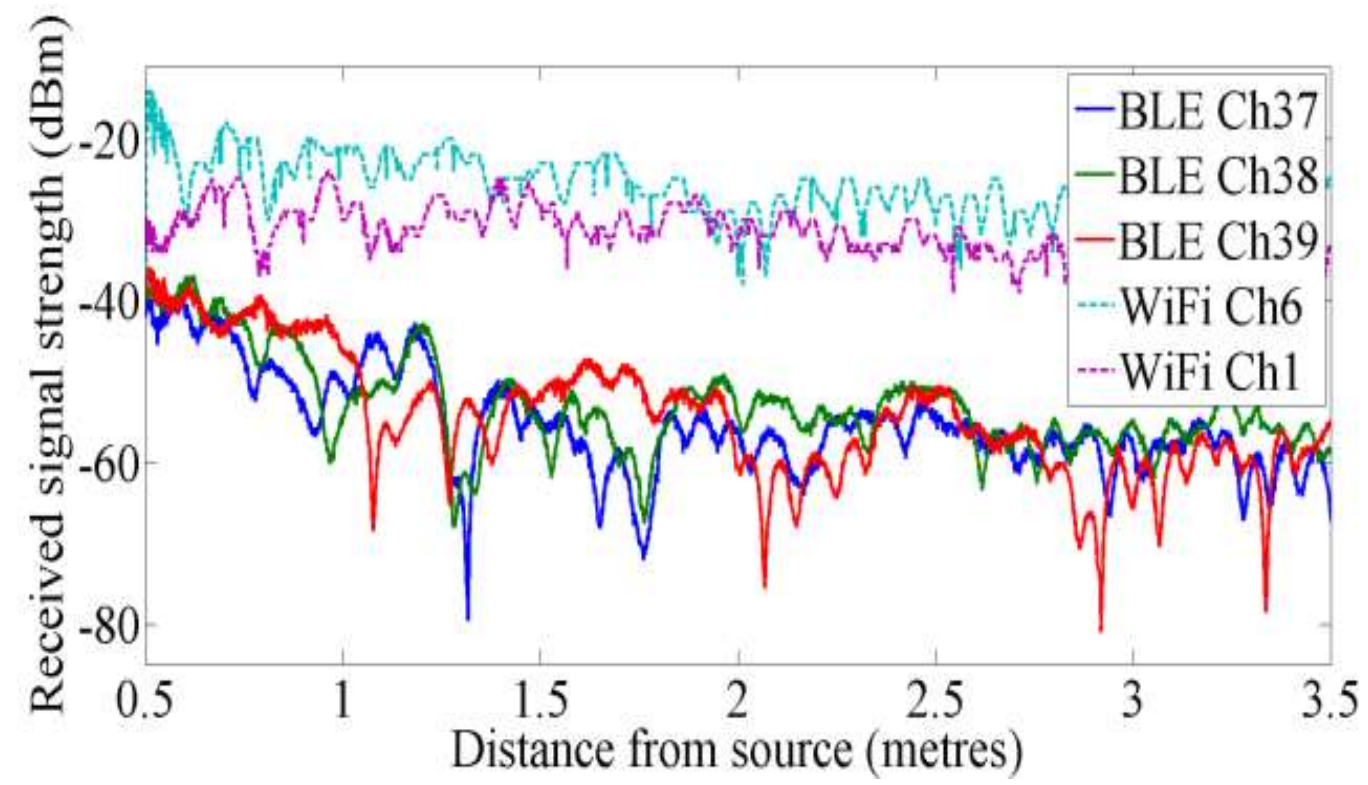

Figure 6. RSSI Comparison Results between Wi-Fi and BLE within 3.5m [12]

Domestically, WiFi based Fingerprinting indoor positioning technique is widely used as the WiFi infra constructed on a national scale thus has high availability. However, it requires many $\mathrm{Wi}-\mathrm{Fi} \mathrm{AP}$ and the increasing cost for installation maintenance, fixation is a huge disadvantage and wireless environment interference such as nearby Wearable equipment makes indoor location positioning harder. BLE is a low electric power based technique, uses beacon without auxiliary equipment and is economic in installation and maintainability. Thus, the researches and realization about indoor positioning technique using BLE get lots of attention in recent times.

\section{Conclusion}

This paper suggested the technique that recognizes motion and position using AHRS based on inertial measurement unit to increase the accuracy of motion and position for the improvement of immersion in full-body-experience VR environment. Position recognition by walk tracking (the relative position recognition method) and the accuracy of the position recognition can improved by removing the cumulative error using BLE fingerprint (absolute position recognition method).

Motion recognition is done by attaching several AHRS devices on the user's body to define coordinates system and based on 9-axis motion information measured by each AHRS devices the user's motion can be recognized. In order to improve the accuracy of motion recognition, user motion data about the position information of each joint corrected by extracting joint angle of body segments. For the implementation of suggesting motion and position recognition techniques, position recognition experiment using acceleration sensors and motion recognition experiment using inertia sensors done.

As a result, the average error of position recognition experiment based on acceleration sensors was $0.25 \mathrm{~m}$, and it decreased to $0.19 \mathrm{~m}$ after the correction through Kalman Filter. The average error of joint angle in the joint angle extracting experiment using two inertia sensors was $3.2^{\circ}$. In the future, the position recognition experiment that combines the location information obtained from this research with the location information acquired through BLE fingerprint technique, and the motion recognition experiment targeting the user's whole body will done. 


\section{Acknowledgments}

This research was supported by Basic Science Research Program through the National Research Foundation of Korea (NRF) funded by the Ministry of Education (NRF2017R1D1A3B03034438).

\section{References}

[1] J. S. Lee, J. A. Noh, S. H. Lim and S. J. Lee, "An Activity Contents Technology Trend Based on Virtual Reality”, ETRI Electronics and Telecommunications Trends, vol. 27, no. 3, (2012) March, pp. 1-73.

[2] J. Y. Jung, J. S. Na, C. W. Lee, G. Y. Lee and J. H. Kim, "Prediction of head movements using neck EMG for VR", Journal of Sensor Science and Technology, vol. 25, no. 5, (2016) May, pp. 365-370.

[3] J. S. Park and Y. C. Seok, "Advertisement Analysis System with Eye Tracking VR HMD(Virtual Reality Head Mounted Display”, Smart media journal, vol. 5, no. 3, (2016) March, pp. 1-5.

[4] A. Y. Kim, W. S. Chae, G. H. Zhang, H. L. Choi, B. S. Kim and J. W. Lee, "Trends Analysis on virtual reality", ETRI Electronics and Telecommunications Trends, vol. 31, no. 4, (2016) April, pp. 23-35.

[5] J. Borenstein, L. Ojeda and S. Kwanmuang, "Heuristic reduction of gyro drift for personnel tracking systems", The Journal of navigation, vol. 62, no. 1, (2009) January, pp. 41-58.

[6] S. H. Fang and T. N. Lin, "Principal component localization in indoor WLAN environments", IEEE Trans. on MobileComputing, vol. 11, no. 1, (2012) January, pp. 100-110.

[7] C. Huang, Z. Liao and L. Zhao, "Synergism of INS and PDR in self-contained pedestrian tracking with a miniature sensor module", IEEE Sensors Journal, vol. 10, no. 8, (2010) August, pp. 1349-1359.

[8] Y. Zhou, C. L. Law, Y. L. Guan and F. Chin, "Indoor elliptical localization based on asynchronous UWB range measurement", IEEE Trans. on Instrumentation and Measurement, vol. 60, no. 1, (2011), pp. 248-257.

[9] J. Park and J. Lee, "A beacon color code scheduling for the localization of multiple robots", IEEE Trans. on Industrial Informatics, vol. 7, no. 3, (2011) March, pp. 467-475.

[10] Oculus. Oculus-Left[Internet]. Available: https://www.oculus.com/rift/.

[11] Y. M. Lim and T. H. Jo, "Development Direction and Implications of Virtual Reality / Augmented Reality Technology", Software Policy \& Research Institute Issue Report, vol. 14, (2017) July, pp. 1-23.

[12] F. Ramsey and R. Harle, "An analysis of the accuracy of bluetooth low energy for indoor positioning applications", Proceedings of the 27th International Technical Meeting of the Satellite Division of the Institute of Navigation (ION GNSS+ 2014), Florida: FL, vol. 812, (2014) September, pp. 201-210.

[13] S. K. Song, "Regulatory Reform Solution of VR Contents Industry based on Simulator", Journal of the Korea Institute of Information and Communication Engineering, vol. 21, no. 11, (2017) November, pp. 2083-2088.

[14] R. J. Conejar and H. K. Kim, "A Review on Mobile Services Secure Mobility Platform”, Journal of Security Engineering, vol. 12, no. 5, (2015) May, pp. 525-532.

[15] J. Kim, "Fast Motion Estimation Based on a Modified Median Operation for Efficient Video Compression", Journal of information and communication convergence engineering, vol. 12, no. 1, (2014), pp. 53-59.

[16] M.-C. Lee, K. Inoue, N. Konishi and J.-J. Lee, "Depth Extraction of Partially Occluded 3D Objects Using Axially Distributed Stereo Image Sensing", Journal of information and communication convergence engineering, vol. 13, no. 4, (2015), pp. 275-279.

[17] Statista, "Number of Active Virtual Reality Users Worldwide from 2014 to 2018," http://www.statista.com/statistics/ 426469/active-virtual-reality-usersworldwide/.

[18] Statista, "Forecast Revenue for Virtual Reality Products Worldwide from 2014 to 2018," http://www.statista.com/ statistics/426276/virtual-reality-revenue-forecastworldwide/.

\section{Authors}

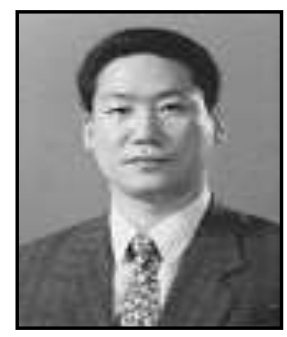

Am-Suk Oh, he received Ph.D degree at the computer engineering of Pusan National University. He is currently with the Department of Media Engineering, Tongmyong University as Professor. His research interests are Healthcare System, Medical Information System, Big Data. 
International Journal of Control and Automation

Vol. 11, No. 10 (2018) 